\title{
肉桂腈电化学还原反应机理
}

\author{
王欢赵淑凤 兰阳春 刘 晓陆嘉星* \\ (华东师范大学化学系, 上海市绿色化学与化工过程绿色化重点实验室, 上海 200062)
}

\begin{abstract}
摘要: 采用循环伏安法研究了肉桂腈在乙腈溶液中的电还原行为, 其在 -1.46 和 $-2.0 \mathrm{~V}$ 处各存在一个还原峰. 第一个峰处恒电位电解得到了线性、环化氢化二聚产物以及苯基戊二腈; 第二个峰处电解得到了饱和二氢还原 产物苯丙腈. 结合循环伏安模拟判定了整个电还原的具体反应机理是肉桂腈通过电化学-电化学-化学-化学 (EECC)反应机理生成苯丙腈, 同时经历自由基-自由基(RR)过程得到线性和环化二聚产物, 肉桂腈还可以与乙腈 的共轭碱反应得到苯基戊二腈. 最终通过循环伏安模拟求得相应反应的动力学常数, 自由基-自由基耦合反应速 率常数为 $10^{4} \mathrm{~L} \cdot \mathrm{mol}^{-1} \cdot \mathrm{s}^{-1}$, 第二个电子转移反应速率常数为 $0.3 \mathrm{~cm} \cdot \mathrm{s}^{-1}$, 其后质子化反应的速率常数为 $10^{5} \mathrm{~s}^{-1}$.
\end{abstract}

关键词：肉桂腈; 电还原; 循环伏安模拟; 反应机理 中图分类号: 0646

\section{Electrochemical Reduction Reaction Mechanism of Cinnamonitrile}

\author{
WANG Huan ZHAO Shu-Feng LAN Yang-Chun LIU Xiao LU Jia-Xing * \\ (Shanghai Key Laboratory of Green Chemistry and Chemical Processes, Department of Chemistry, \\ East China Normal University, Shanghai 200062, P. R. China)
}

\begin{abstract}
The electroreduction of cinnamonitrile in $\mathrm{MeCN}$ solution was studied using cyclic voltammetry, with two reduction peaks at -1.46 and $-2.0 \mathrm{~V}$. Linear, cyclic hydrodimers, and 3-phenyl pentanedinitrile were synthesized at the first peak, while additional saturated hydro product was synthesized at the second peak. The reduction mechanism was proposed based on the experimental and cyclic voltammogram simulation results. Linear and cyclic hydrodimers were synthesized by a radical-radical (RR) route while a saturated hydro product was obtained via an electrochemicalelectrochemical-chemical-chemical (EECC) process. 3-Phenyl pentanedinitrile was synthesized by the reaction of cinnamonitrile with the conjugate base of $\mathrm{MeCN}$. Finally, the dynamic constants were calculated using cyclic voltammogram simulation. Result indicated that the rate constant of the RR reaction was found to be $10^{4} \mathrm{~L} \cdot \mathrm{mol}^{-1} \cdot \mathrm{s}^{-1}$, and the rate constant of the second electron transfer reaction was $0.3 \mathrm{~cm} \cdot \mathrm{s}^{-1}$, while the rate constant of the subsequent proton reaction was $10^{5} \mathrm{~s}^{-1}$.
\end{abstract}

Key Words: Cinnamonitrile; Electroreduction; Cyclic voltammogram simulation; Reaction mechanism

电还原由吸电子基团(如一 $\mathrm{CN},-\mathrm{CHO},-\mathrm{COR}$, $-\mathrm{COOH}$ 和一 $\mathrm{COOR}$ 等)活化的烯烃是一类非常重 要的有机电合成反应. 早在 20 世纪 60 年代中期, 美 国孟山都公司的贝泽教授就研究了丙烯腈的电解还 原二聚反应 ${ }^{[1-2]}$, 并将其大规模工业化, 成为生产己
二腈的重要手段. 从合成机理 ${ }^{[3-4]}$ 、立体构型 ${ }^{[5]}$ 、分子 结构密度函数理论计算 ${ }^{[6]}$ 等角度, 肉桂酸酯 $(\mathrm{Ph}-$ $\mathrm{CH}=\mathrm{CH}-\mathrm{COOR}$ )的电还原反应已被广泛研究, 其 经历的电子还原反应主要生成反式环化氢化二聚产 物, 同时得到少量内消旋线性氢化二聚产物, 而经过

Received: August 20, 2009; Revised: October 29, 2009; Published on Web: December 29, 2009.

*Corresponding author. Email: jxlu@chem.ecnu.edu.cn; Tel: +86-21-62233491.

The project was supported by the National Natural Science Foundation of China (20973065), Basic Research in Natural Science Issued by Shanghai Municipal Committee of Science, China (08dj1400100), and Shanghai Leading Academic Discipline Project, China (B409).

国家自然科学基金(20973065), 上海市科委重大项目(08dj1400100)和上海市物理化学重点学科(B409)资助

C. Editorial office of Acta Physico-Chimica Sinica 
两电子还原可以得到苯丙酸酯. 另外, 在饱和的 $\mathrm{CO}_{2}$ 气氛下电还原肉桂酸酯可以进一步合成各种多羧基 芳香化合物 ${ }^{[7-9]}$. 同样, $\alpha, \beta$-不饱和酮 $(\mathrm{Ph}-\mathrm{CH}=$ $\mathrm{CH}-\mathrm{CO}-\mathrm{R})$ 的电还原反应也被深人考察 ${ }^{[10-12]}$. 然而 具有类似结构的肉桂腈的电还原反应却鲜有报 道 ${ }^{[13]}$. 另一方面, 传统的电还原反应的机理主要是通 过循环伏安法、计时电流电量法 ${ }^{[14]}$ 、原位红外法 ${ }^{[15]}$ 等 $^{-1}$ 进行推测分析得到的. 本文则采用循环伏安模拟的 方法对肉桂腈在乙腈中的电还原反应机理进行分析 判断. 首先通过循环伏安法考察了其电化学行为特 征, 接着通过恒电位电解分别在两个峰处还原得到 相应的产物, 由此推测反应的机理; 然后运用循环 伏安模拟来判定具体的反应过程, 并求得相应反应 的动力学常数.

\section{1 实验部分}

\section{1 仪器与试剂}

CHI650 型电化学工作站 (上海辰华仪器公司), HP6890/5973N GC-MS 色质联用仪(美国 Agilent HP 公司), DigiSim(version 3.03, 美国 BAS 公司)循环伏 安模拟软件 ${ }^{[16}$. 玻碳( GC) 电极(天津艾达科技恒晟科 技发展有限公司), 螺旋铂丝电极 (自制), $\mathrm{Ag} / \mathrm{AgI} / \mathrm{I}^{-}$参 比电极 (上海辰华仪器公司).

氮气 $\left(\mathrm{N}_{2}\right.$, 上海都茂爱净气有限公司, 纯度 99.999\%), 肉桂腈(武汉远城科技发展有限公司, $98 \%$ ), 四乙基四氟嗍酸铵 $\left(\mathrm{Et}_{4} \mathrm{NBF}_{4}\right)$ 由 $\mathrm{Et}_{4} \mathrm{NOH}$ 和 $\mathrm{HBF}_{4}$ 中和结晶、重结晶纯化制备 ${ }^{[17-18]}$, 并经过真空干燥箱 干燥. 其余试剂均为分析纯. 实验用水为蒸馏水.

\section{2 循环伏安实验}

在 $25{ }^{\circ} \mathrm{C}$ 下, 一室型电解池中加人 $10 \mathrm{~mL}$ $\mathrm{MeCN}-0.1 \mathrm{~mol} \cdot \mathrm{L}^{-1} \mathrm{Et}_{4} \mathrm{NBF}_{4}-2 \mathrm{mmol} \cdot \mathrm{L}^{-1}$ 肉桂腈混合 液, 通 $\mathrm{N}_{2} 30 \mathrm{~min}$ 除去溶液中的溶解氧, 以 $\mathrm{GC}$ 为工 作电极, $\mathrm{Pt}$ 为辅助电极, $\mathrm{Ag} / \mathrm{AgI} / \mathrm{I}^{-}$为参比电极, 用电 化学工作站进行循环伏安扫描. 并用循环伏安模拟 (DigiSim)对循环伏安图进行模拟拟合.

\section{3 恒电位电解实验}

在 $25{ }^{\circ} \mathrm{C}$ 下, 一室型电解池中加人 $10 \mathrm{~mL}$ $\mathrm{MeCN}-0.1 \mathrm{~mol} \cdot \mathrm{L}^{-1} \mathrm{Et}_{4} \mathrm{NBF}_{4}-0.1 \mathrm{~mol} \cdot \mathrm{L}^{-1}$ 肉桂腈混合 液, 通 $\mathrm{N}_{2} 30 \mathrm{~min}$ 除去溶液中的溶解氧, 以不锈钢为 工作电极, 镁为牺牲阳极, $\mathrm{Ag} / \mathrm{AgI} / \mathrm{I}^{-}$为参比电极, 用 电化学工作站进行恒电位电解. 电解结束后, 用蒸馏 水稀释、乙醚萃取. 醚层通过 GC-MS 定性定量检测.

\section{2 结果与讨论}

\section{1 肉桂腈的循环伏安行为及恒电位产物}

首先利用循环伏安图研究肉桂腈在乙腈溶液中 的电还原行为 (见图1). 在玻碳 (GC) 电极上, 当扫描 速度为 $0.1 \mathrm{~V} \cdot \mathrm{s}^{-1}$ 时, 肉桂腈在 $\mathrm{C}_{1}\left(E_{\mathrm{pl}}=-1.46 \mathrm{~V}(\mathrm{vs} \mathrm{Ag} /\right.$ $\left.\mathrm{AgI} / \mathrm{I}^{-}\right)$)处有一还原峰, 在电位更低的 $\mathrm{C}_{2}\left(E_{\mathrm{p} 2}=-2.0 \mathrm{~V}\right.$ (vs $\left.\mathrm{Ag} / \mathrm{AgI} / \mathrm{I}^{-}\right)$)处还有一个不可逆的还原峰. 这两个 还原峰对应于肉桂腈苯基和腈基之间的碳碳双键还 原的两个电子转移过程.

为了进一步了解肉桂腈在乙腈溶液中电还原的 具体过程, 分别在两个还原峰处对该体系进行恒电 位电解, 具体结果如 Scheme 1 所示. 在 $-1.6 \mathrm{~V}(\mathrm{vs}$ $\left.\mathrm{Ag} / \mathrm{AgI} / \mathrm{I}^{-}\right)$处对肉桂腈 1 电还原, 主要得到两种氢化 二聚物, 线性二聚 2(48\%) 和环化二聚 3(42\%), 以及 还有少量的戍二腈衍生物 4(10\%). 而在-2.1 V ( v s $\mathrm{Ag} / \mathrm{AgI} / \mathrm{I}^{-}$)处电还原, 除了得到上述三种产物(36\%的 2, 33\%的 3 和 $29 \%$ 的 4) 外, 还得到了饱和的二氢还 原产物苯丙腈 $\mathbf{5}(2 \%)$.

二聚产物是碳碳双键得到一个电子后通过后续 化学反应生成的, 而饱和二氢还原产物是碳碳双键 得到两个电子完全还原的结果. 这符合上述循环伏 安行为的特征, 即肉桂腈在第一个还原峰处得到一 个电子, 然后经过一系列的后续化学反应生成二聚 产物; 或者是再继续得到一个电子完全还原后生成 饱和二氢产物.

\section{2 肉桂腈电还原可能的反应历程分析}

\subsection{1 苯丙腈合成机理}

肉桂腈完全还原成苯丙腈, 整个过程需要得到 两个电子和两个质子. 可能的过程有两个: EECC(电

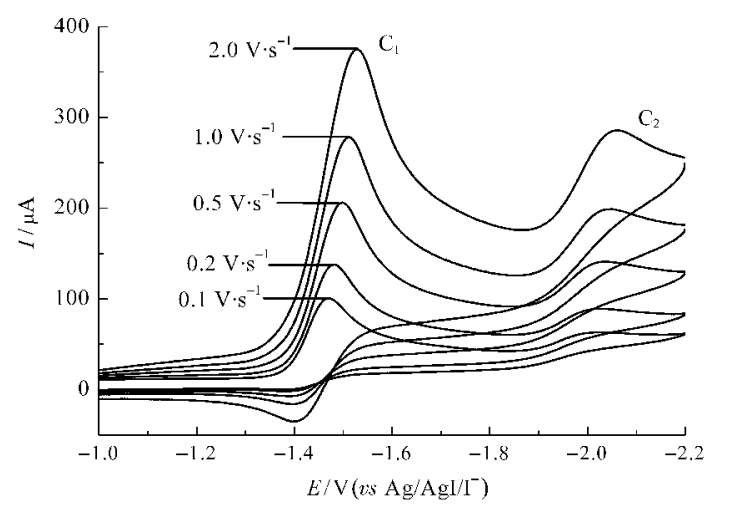

图 1 不同扫描速率下肉桂腈在乙腈溶液中的循环伏安图 Fig.1 Cyclic voltammograms of cinnamonitrile with different scan rates in $\mathrm{MeCN}$ solution recorded at GC electrode in $\mathrm{MeCN}-0.1 \mathrm{~mol} \cdot \mathrm{L}^{-1} \mathrm{Et}_{4} \mathrm{NBF}_{4}$ for $2 \mathrm{mmol} \cdot \mathrm{L}^{-1}$ cinnamonitrile at $25{ }^{\circ} \mathrm{C}$ with scan rates from $0.1 \mathrm{~V} \cdot \mathrm{s}^{-1}$ to $2.0 \mathrm{~V} \cdot \mathrm{s}^{-1}$ 


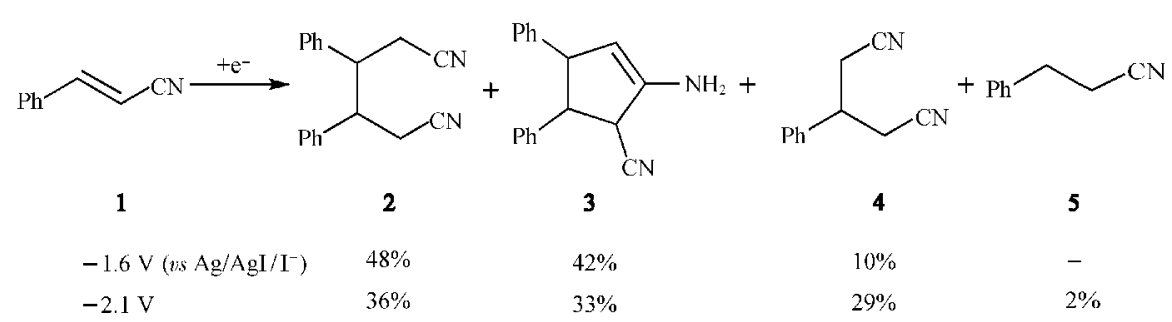

Scheme 1 Potentialstatic electrolysis

化学-电化学-化学-化学)和 ECEC(电化学-化学-电 化学-化学). 对于 EECC 机理(Scheme 2), 肉桂腈 $\mathbf{1}$ 首先在电极表面得到一个电子, 生成其碳负离子自 由基 $\mathbf{6}$, 然后碳负离子自由基中间体 6 继续得到一 个电子, 生成二价碳负离子 7 , 接着二价碳负离子中 间体 7 得到两个质子, 最后生成饱和二氢还原产物 苯丙腈 5. 而对于 ECEC 机理(Scheme 3), 第一步也 是通过电子转移反应得到碳负离子自由基 6 , 但第 二步是碳负离子自由基 6 质子化的均相化学反应, 接着上面生成的自由基中间体 8 再得到一个电子, 生成碳负离子中间体 $\mathbf{9}$, 最后其再与一个质子结合, 生成苯丙腈 $\mathbf{5}$.

\subsection{2 二聚产物生成机理}

肉桂腈还原得到线性二聚产物也有两种可能的 过程. RR(自由基-自由基)机理: 两个得一电子生成

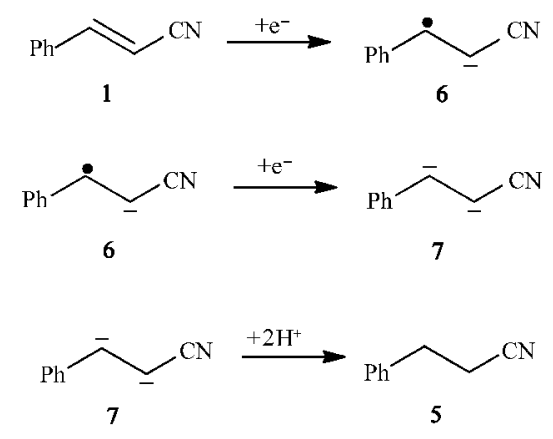

El

Scheme 2 EECC mechanism

E: electrochemical; C: chemical

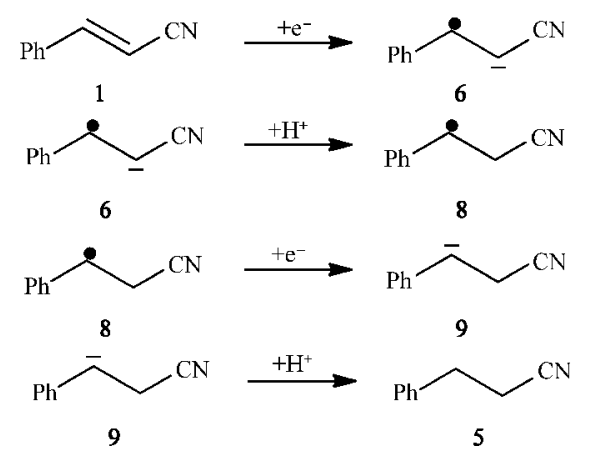

Scheme 3 ECEC mechanism

$\mathrm{CC}$

El

C2
的碳负离子自由基 6 通过偶合, 产生二价碳负离子 10 , 然后该中间体再得到两个质子合成线性二聚产 物 2(Scheme 4). RS(自由基-底物)机理 ${ }^{[19]}$ : 由碳负离 子自由基 6 进攻未还原的底物 1 , 生成新的碳负离 子自由基中间体 11; 接着该中间体 $\mathbf{1 1}$ 再与一个碳 负离子自由基 6 发生均相电子转移反应, 生成二价 碳负离子中间体 10; 最后通过质子化得到二聚产物 (Scheme 5).

需要补充说明的一点是, 由文献 $[3-5,20]$ 可知, 环化二聚产物的生成是由于得到一个质子后的中间

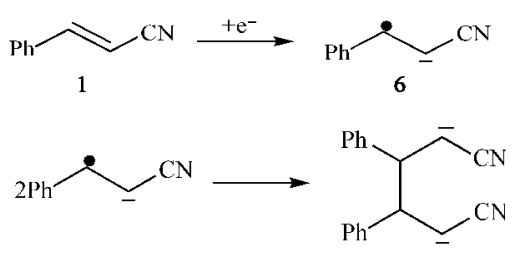

6

10<smiles>N#CCC(c1ccccc1)C(CC#N)C(CC#N)c1ccccc1</smiles>

Scheme 4 RR mechanism $\mathrm{R}$ : radical<smiles>N#CCCPC#CCC=Cc1ccccc1</smiles><smiles>N#CCC(c1ccccc1)C(CC#N)C(C#N)c1ccccc1</smiles>

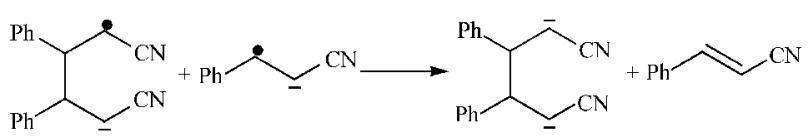

11<smiles>N#CCC(c1ccccc1)C(CC#N)c1ccccc1</smiles><smiles></smiles>

10

1

Scheme 5 RS mechanism S: substrate 
<smiles>CCC(c1ccccc1)C(CC#N)c1ccccc1</smiles>

10

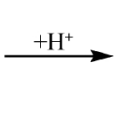<smiles>N#CCCC1CCCCC1c1ccccc1</smiles>

12<smiles>C[18CH]</smiles><smiles>N#CC1C(N)=CC(c2ccccc2)C1c1ccccc1</smiles>

Scheme 6 Dieckmann condensation

体 12 容易发生 Dieckmann 缩合反应(Scheme 6), 而 进行环化. 也就是说两种二聚产物前面的反应历程 是一致的.

\section{3 具体反应机理的判定}

根据 2.2.1 节中对苯丙腈合成机理的分析可知, 在第一个电子得失后跟着的是一个化学反应(ECEC 机理)或一个电化学反应(EECC 机理). 先假设后面 跟的是均相化学反应, 且反应速度很快, 碳负离子自 由基 6 在电极上一生成立即转变为自由基中间体 8, 那么在回扫的过程中则无法检测到相应的氧化 峰. 由图 1 对扫描速率的考察发现, 随着扫描速率的 增大, 第一个还原峰相对应的氧化峰越来越明显. 该 现象也说明在第一个异相电子转移反应后紧跟着比 较慢的均相化学反应. 由此可以推测, 如果 ECEC 是生成苯丙腈的机理的话, 第一个化学反应(C)必须 是一个速率比较慢的反应. 那么, 由于均相化学反应 的速率较慢, 随着扫速的增大, 扫描过程所需的时间 也越短, 给均相化学反应的时间也越短, 那么在具体 的扫描过程中生成的自由基中间体的量也会相应减 少, 从而使第二个峰的相应峰电流比例下降(如根据 ECEC 机理模拟的循环伏安图如图 2 所示). 而实际 测量的是, 随着扫速的增大, 第二个还原峰的峰电流 比例 $\left(I_{\mathrm{pc} 2} / I_{\mathrm{pc1}}\right)$ 逐渐增大(如图 1 和表 1 所示).上述分 析说明, 在生成苯丙腈的过程中, 第一个电子转移反 应后不可能紧跟着 ECEC 机理中所假设的质子化 过程, 而只可能紧接着进行第二个电子转移反应, 所 以应该经历 EECC 机理.

既然饱和二氢还原产物的合成是经历 EECC 机理, 那么如果没到第二个电子转移的电位, 该反 应就不会发生. 也就是说当回扫电位由-2.2 V 改为 $-1.8 \mathrm{~V}$ 后(见图 3), 得到的循环伏安图只与二聚反 应有关, 从而可以判断该反应的机理. 由表 2 可知, 当扫描速率为 $0.1 \mathrm{~V} \cdot \mathrm{s}^{-1}$ 时, 半峰宽值为 $50 \mathrm{mV}$, 比 在纯动力学控制下 $\mathrm{RS}$ 机理的理论值 $(58.3 \mathrm{mV}$ )要 小. 随着扫描速率的减小, 峰形越来越趋于纯动力学 控制特征. 在最小扫速 $0.01 \mathrm{~V} \cdot \mathrm{s}^{-1}$ 下, 半峰宽值为 $45 \mathrm{mV}$, 接近纯动力学条件下 $\mathrm{RR}$ 机理的极限值
$(38.8 \mathrm{mV})$. 同时, 由上述数据还可以求出该体系的 $-\mathrm{d} E_{\mathrm{p}} / \mathrm{d}(\lg v)$ 为 $23.3 \mathrm{mV}$, 接近纯动力学控制下 $\mathrm{RR}$ 机理 的极限值 $(19.7 \mathrm{mV})$, 而 $\mathrm{RS}$ 机理的理论值为 $29.6 \mathrm{mV}$. 值得注意的是, 慢的电子转移速率和未补偿的 $i R$ 降 会使得半峰宽值变大, 从而使数据产生偏差. 实际测 量中发现该体系的未补偿电阻为 $100 \Omega$ 左右, 后面 的循环伏安模拟算出值为 $110 \Omega$, 这就解释了实验 测得的半峰宽值比理论值偏大. 由此我们可以推断, 肉桂腈在乙腈溶液中二聚的速控步骤是 RR 机理

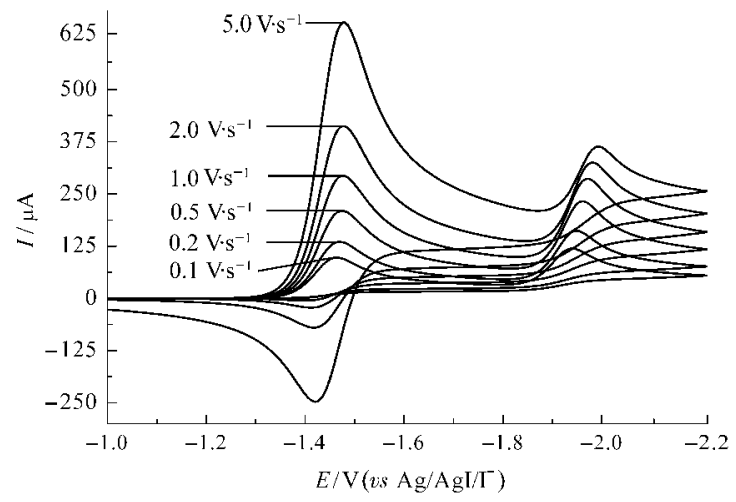

图 2 模拟 ECEC 机理还原的循环伏安图

Fig.2 Cyclic voltammograms for simulated ECEC mechanism

The simulated voltammograms were calculated for ECEC process (as shown in Scheme 3). Symbols: digital simulation with standard potential for El, $E_{1}^{0}=-1.45 \mathrm{~V}$; electron transfer coefficient for El, $\alpha_{1}=0.5$; heterogeneous charge-transfer rate constant for El, $k_{\mathrm{s}, 1}=10^{4} \mathrm{~cm} \cdot \mathrm{s}^{-1}$; standard potential for $\mathrm{E} 2, E_{2}^{0}=-2.05 \mathrm{~V}$; electron transfer coefficient for E2,$\alpha_{2}=0.5$, heterogeneous charge-transfer rate constant for $\mathrm{E} 2$ (Scheme 3 ), $k_{\mathrm{s}, 2}=10^{4} \mathrm{~cm} \cdot \mathrm{s}^{-1}$, equilibrium constant for $\mathrm{Cl}, K_{\mathrm{Cl}}=10^{6}$; rate constant for $\mathrm{Cl}, k_{\mathrm{fCl}}=5 \mathrm{~s}^{-1}$ : equilibrium constant for $\mathrm{C} 2, K_{\mathrm{C} 2}=10^{6}$, rate constant for $\mathrm{C} 2, k_{\mathrm{fC}}=10^{5} \mathrm{~s}^{-1}$; electrode area $A=0.07 \mathrm{~cm}^{2}$; diffusion coefficients for all species,

$D_{\text {all species }}=1 \times 10^{-5} \mathrm{~cm}^{2} \cdot \mathrm{s}^{-1} ; T=298 \mathrm{~K}$; scan rate: $0.1-5.0 \mathrm{~V} \cdot \mathrm{s}^{-1}$

表 1 扫描速率对两个还原峰峰电流比的影响

Table 1 Effect of scan rate on the two reduction peak current ratio

\begin{tabular}{cccc}
\hline$v /\left({\left.\mathrm{V} \cdot \mathrm{s}^{-1}\right)}^{c}\right.$ & $10^{4} I_{\mathrm{pc} 1} / \mathrm{A}$ & $10^{5} I_{\mathrm{pc} 2} / \mathrm{A}$ & $I_{\mathrm{pc} 2} / I_{\mathrm{pc} 1}$ \\
\hline 2.0 & 3.158 & 10.98 & 0.3477 \\
1.0 & 2.385 & 7.279 & 0.3052 \\
0.5 & 1.786 & 4.892 & 0.2739 \\
0.2 & 1.187 & 2.872 & 0.2420 \\
0.1 & 0.8794 & 2.035 & 0.2314 \\
\hline
\end{tabular}




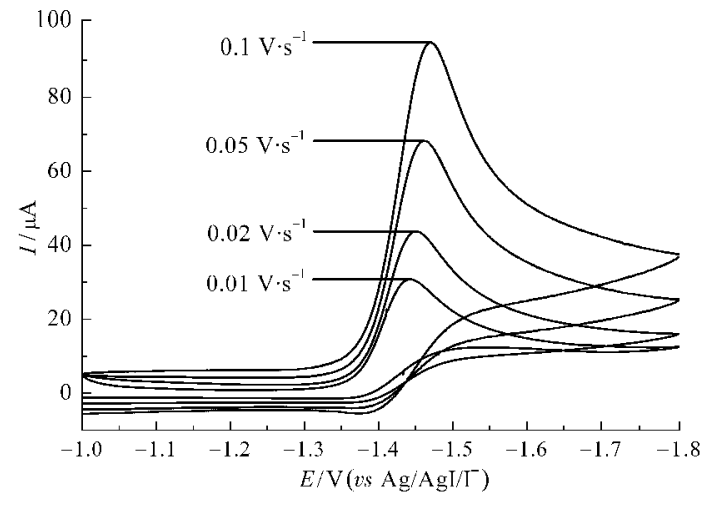

图 3 不同扫描速度下肉桂腈在 $\mathrm{MeCN}$ 中第一个还原峰的 循环伏安图

Fig.3 Cyclic voltammograms of cinnamonitrile for the first reduction peak with different scan rates in MeCN solution

recorded at GC electrode in $\mathrm{MeCN}-0.1 \mathrm{~mol} \mathrm{~L}^{-1} \mathrm{Et}_{4} \mathrm{NBF}_{4}$ for $2 \mathrm{mmol} \cdot \mathrm{L}^{-1}$ cinnamonitrile at $25{ }^{\circ} \mathrm{C}$ with scan rates from 0.01 to $0.1 \mathrm{~V} \cdot \mathrm{s}^{-1}$

\section{表 2 肉桂腈循环伏安数据}

Table 2 Cyclic voltammetric results for cinnamonitrile

\begin{tabular}{ccc}
\hline$v /\left(\mathrm{V} \cdot \mathrm{s}^{-1}\right)$ & $\left(E_{\mathrm{p}}-E_{\mathrm{p} / 2}\right) / \mathrm{mV}$ & $\left(-\mathrm{d} E_{\mathrm{p}} / \mathrm{d}(\lg v)\right) / \mathrm{mV}$ \\
\hline 0.01 & 45 & 23.3 \\
0.02 & 45 & \\
0.05 & 47 & \\
0.1 & 50 & \\
\hline
\end{tabular}

过程.

另外, 戊二腈衍生物 4 的生成主要是因为 $\mathrm{MeCN}$ 的酸性比较大, 其共轭碱 $\mathrm{CNCH}_{2}^{-}$可以与未反 应的肉桂腈 1 发生 Michael 加成, 再进一步质子化 得到腈甲基化的产物 4(如 Scheme 7).

\section{4 循环伏安图的模拟}

由上面的分析可知, 肉桂腈在乙腈中第一个还 原峰只包含其二聚反应的特征信息, 因而我们可以 通过对该循环伏安图形的模拟得到相应的反应动力 学参数, 实验及模拟图形如图 4 所示.

根据还原峰倾斜程度, 模拟的该体系的未补偿 电阻为 $110 \Omega$; 由氧化还原峰的比例模拟得, RR 反 应的速率常数为 $10^{4} \mathrm{~L} \cdot \mathrm{mol}^{-1} \cdot \mathrm{s}^{-1}$; 根据峰电流的大小

$$
\mathrm{Ph} \curvearrowright-\mathrm{CN}+\mathrm{CNCH}_{2}^{-} \longrightarrow
$$

1<smiles>N#CCPC(CC#N)CC#N</smiles>

Scheme 7 Michael addition
模拟得, 肉桂腈在 $\mathrm{MeCN}$ 中的扩散系数为 $4.72 \times 10^{-5}$ $\mathrm{cm}^{2} \cdot \mathrm{s}^{-1}$.

运用第一个还原峰的模拟数据再对整个循环伏 安图进行模拟, 以求得生成苯丙腈的相应动力学参 数, 实验及模拟图形如图 5 所示. 由第二个还原峰的

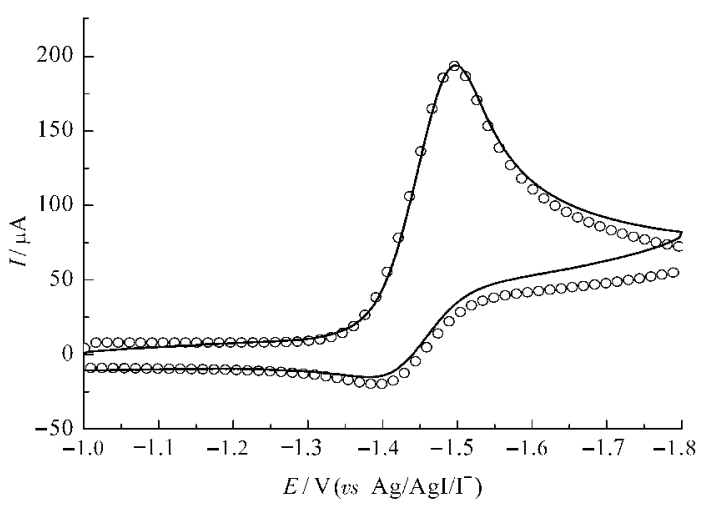

图 4 肉桂腈在 $\mathrm{MeCN}$ 中第一个电子转移反应的实验(-) 及其模拟 $(\bigcirc)$ 循环伏安图对比

Fig.4 Comparison of experimental (-) and simulated $(\bigcirc)$ cyclic voltammograms for the first electron transfer reaction of cinnamonitrile in $\mathrm{MeCN}$ solution The simulated voltammograms were calculated for RR process (as shown in Scheme 4). Symbols: digital simulation with $E_{1}^{0}=-1.45 \mathrm{~V} ; \alpha_{1}=0.5 ; k_{\mathrm{s}, 1}=10^{4} \mathrm{~cm} \cdot \mathrm{s}^{-1} ;$ equilibrium constant for RR, $K_{\mathrm{RR}}=10^{6} ;$ rate constant for RR, $k_{\mathrm{RRR}}=10^{4} \mathrm{~L} \cdot \mathrm{mol}^{-1} \cdot \mathrm{s}^{-1} ; A=0.07 \mathrm{~cm}^{2}$; $D=4.72 \times 10^{-5} \mathrm{~cm}^{2} \cdot \mathrm{s}^{-1} ; C=2 \mathrm{mmol} \cdot \mathrm{L}^{-1} ;$ uncompensated resistance, $R=110 \Omega$; double-layer capacitance, $C_{\mathrm{d}}=1.6 \times 10^{-5} \mathrm{~F}$; $T=298 \mathrm{~K}$; scan rate: $0.5 \mathrm{~V} \cdot \mathrm{s}^{-1}$

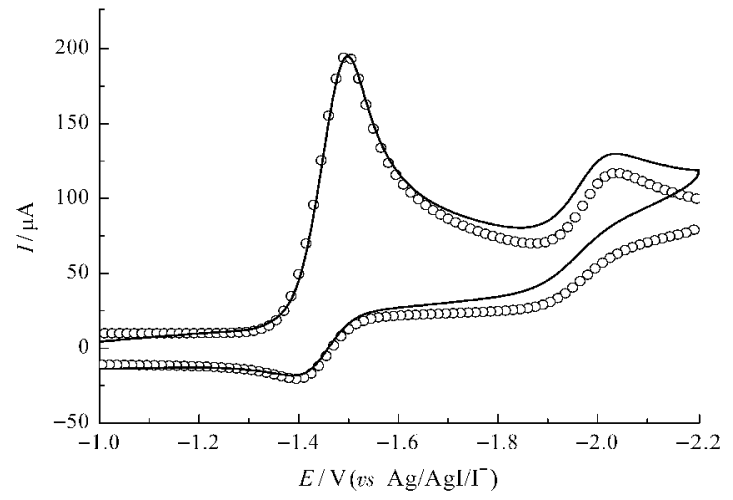

图 5 肉桂腈在 $\mathrm{MeCN}$ 中整个还原过程的实验(-)及其模 拟( ○)循环伏安图对比

Fig.5 Comparison of experimental (-) and simulated $(\bigcirc)$ cyclic voltammograms for the reduction of cinnamonitrile in $\mathrm{MeCN}$ solution

The simulated voltammograms was calculated for EECC \& RR process (as shown in Schemes $2 \& 4$ ). Symbols: digital simulation with $E_{1}^{0}=-1.45 \mathrm{~V}, \alpha_{1}=0.5, k_{\mathrm{s}, 1}=10^{4} \mathrm{~cm} \cdot \mathrm{s}^{-1}, E_{2}^{0}=-2.05 \mathrm{~V}, \alpha_{2}=0.5$,

$k_{\mathrm{s}, 2}=0.3 \mathrm{~cm} \cdot \mathrm{s}^{-1}, K_{\mathrm{RR}}=10^{6}, k_{\mathrm{RRR}}=10^{4} \mathrm{~L} \cdot \mathrm{mol}^{-1} \cdot \mathrm{s}^{-1}, K_{\mathrm{C}}=10^{6}, k_{\mathrm{fC}}=10^{5} \mathrm{~s}^{-1}$, $A=0.07 \mathrm{~cm}^{2}, D=4.72 \times 10^{-5} \mathrm{~cm}^{2} \cdot \mathrm{s}^{-1}, C=2 \mathrm{mmol} \cdot \mathrm{L}^{-1}, R=110 \Omega$, $C_{\mathrm{dl}}=2 \times 10^{-5} \mathrm{~F}, T=298 \mathrm{~K}$; scan rate: $0.5 \mathrm{~V} \cdot \mathrm{s}^{-1}$ 
峰形模拟得: 第二个电子转移反应的速率常数为 0.3 $\mathrm{cm} \cdot \mathrm{s}^{-1}$, 其后质子化反应的速率常数为 $10^{5} \mathrm{~s}^{-1}$.

\section{3 结 论}

采用循环伏安法和恒电位电解法考察了肉桂腈 在乙腈溶液中的电还原反应, 并结合循环伏安的模 拟计算推断了整个电还原反应机理. 肉桂腈在乙腈 溶液中呈现两个还原峰, 随着扫描速率的增大, 第一 个还原峰相对应的氧化峰越来越明显, 表明其后紧 跟着反应速率比较慢的均相化学反应; 而第二个还 原峰一直保持不可逆特征. 通过研究推得了整个反 应机理: 肉桂腈经历 EECC 过程合成苯丙腈, 通过 $\mathrm{RR}$ 历程生成线性和环化氢化二聚产物, 与乙腈的 共轭碱反应生成苯基戊二腈. 由循环伏安行为模拟 求得自由基-自由基耦合反应速率常数为 $10^{4} \mathrm{~L}$ $\mathrm{mol}^{-1} \cdot \mathrm{s}^{-1}$; 第二个电子转移反应速率常数为 $0.3 \mathrm{~cm}$. $\mathrm{s}^{-1}$, 其后质子化反应的速率常数为 $10^{5} \mathrm{~s}^{-1}$.

\section{References}

1 Baizer, M. M. Tetrahedron Lett., 1963, 4: 973

2 Baizer, M. M. J. Electrochem. Soc., 1964, 111: 215

3 Fussing, I.; Gullu, M.; Hammerich, O.; Hussain, A.; Nielsen, M. F.; Utley, J. H. P. J. Chem. Soc. Perkin Trans. 2, 1996, (4): 649

4 Fussing, I.; Hammerich, O.; Hussain, A.; Nielsen, M. F.; Utley, J. H. P. Acta Chem. Scand., 1998, 52: 328

5 Kise, N.; Iitaka, S.; Iwasaki, K.; Ueda, N. J. Org. Chem., 2002, 67: 8305
6 Kise, N. J. Org. Chem., 2006, 71: 9203

7 Wang, H.; Du, Y. F.; Lin, M. Y.; Zhang, K.; Lu, J. X. Chin. J. Chem., 2008, 26: 1745

8 Wang, H.; Zhang, G. R.; Liu, Y. Z.; Luo, Y. W.; Lu, J. X. Electrochem. Commun., 2007, 9: 2235

9 Wang, H.; Zhang, K.; Liu, Y. Z.; Lin, M. Y.; Lu, J. X. Tetrahedron, 2008, 64: 314

10 Utley, J. H. P.; Smith, C. Z.; Motevalli, M. J. Chem. Soc. Perkin Trans. 2, 2000, (5): 1053

11 Kise, N.; Kitagishi, Y.; Ueda, N. J. Org. Chem., 2004, 69: 959

12 Lin, M. Y.; Wang, H.; Zhang, A. J.; Zhang, G. R.; Lu, J. X. Chin. J. Org. Chem., 2008, 28: 1572 [林美玉, 王 欢, 张爱健, 张贵荣, 陆嘉星. 有机化学, 2008, 28: 1572]

13 Wang, H.; Lin, M. Y.; Zhang, K.; Li, S. J.; Lu, J. X. Aust. J. Chem., 2008, 61: 526

14 Zhao, P.; Fang, H. J.; Xue, T.; Qi, X. M.; Lu, J. X. Acta Phys. Chim. Sin., 2005, 21: 1235 [赵 鹏, 方慧娾, 薛 腾, 元西敏, 陆嘉星. 物理化学学报, 2005, 21: 1235]

15 Li, M. C.; Wu, H. F.; Hu, J. Q.; Ma, C. A. Acta Phys. -Chim. Sin., 2008, 24: 1937 [李美超, 吴海峰, 胡佳琦, 马淳安. 物理化学学 报, 2008, 24: 1937]

16 Rudolph, M.; Reddy, D. P.; Feldberg, S. W. Anal. Chem., 1994, 66: A 589

17 Sawyer, D. T.; Robert Jr., J. L. Experimental electrochemistry for chemists. New York: John Wiley \& Sons Inc., 1974

18 Zoski, C. G. Handbook of electrochemistry. Amsterdam: Elsevier, 2007

19 Lund, H.; Hammerich, O. Organic electrochemistry. 4th ed. New York: Marcel Dekker Inc., 2001: 119-120

20 Utley, J. H. P.; Gullu, M.; Motevalli, M. J. Chem. Soc. Perkin Trans. 1, 1995, (15): 1961 